\title{
ESTRUTURA E DINÂMICA DE FLORESTA DE VÁRZEA NO ESTUÁRIO AMAZÔNICO NO ESTADO DO AMAPÁ
}

\author{
José Antonio Leite de Queiroz*, Sebastião do Amaral Machado** \\ Roberto Tuyoshi Hosokawa**, Ivan Crespo da Silva** \\ *Eng. Florestal, M.Sc., Doutorando em Engenharia Florestal, UFPR, Embrapa - leite.queiroz@terra.com.br \\ **Eng. Florestal, Dr., Depto. de Ciências Florestais, UFPR - sammac@floresta.ufpr.br \\ roberto.hosokawa@pesquisador.cnpq.br - ivancrespo@ufpr.br \\ Recebido para publicação: 17/11/2006 - Aceito para publicação: 01/02/2007
}

\begin{abstract}
Resumo
O presente trabalho de pesquisa teve como objetivo principal analisar a estrutura e a dinâmica de floresta de várzea estuarina amazônica, no município de Mazagão, estado do Amapá, e área próxima, com a qual mantém intenso relacionamento socioeconômico. Em 2000, foram instaladas e medidas três parcelas amostrais permanentes de $100 \times 100 \mathrm{~m}$, divididas em dez subparcelas de $20 \times 50 \mathrm{~m}$ cada uma. Em 2005, foi realizada nova mensuração nessas unidades, quando foram avaliados as alterações na estrutura horizontal, o incremento em DAP, a mortalidade e o ingresso de todas as árvores com $\mathrm{CAP} \geq 15 \mathrm{~cm}$. No Furo do Mazagão não ocorreram alterações em relação ao número de espécies (52) e de famílias (27) no período de 2000 a 2005. No rio Maniva, em 2000, havia 48 espécies de 24 famílias; em 2005 surgiu uma espécie e uma família novas. A área basal aumentou em $4,96 \mathrm{~m}^{2} / \mathrm{ha}$. No rio Mutuacá, em 2000, havia 76 espécies de 32 famílias; em 2005 surgiram cinco espécies novas, duas desapareceram e o número de famílias se manteve o mesmo. A densidade absoluta diminuiu em 58 árvores por hectare. A área basal diminuiu em $3,05 \mathrm{~m}^{2} / \mathrm{ha}$.
\end{abstract}

Palavras-chave: Fitossociologia; estuário amazônico; dinâmica florestal.

\begin{abstract}
Structure and dynamics of floodplain forest in the amazonian estuary in the Amapa state. The present study was developed aiming to evaluate the structure and the dynamics of Amazonian estuarine floodplain forest, in the district of Mazagão, Amapá state and in nearby area with which it keeps intense partner-economic relationship. In 2000 three permanent sample plots were installed with size $100 \times 100$ m, divided in ten sub-plots with $20 \times 50 \mathrm{~m}$, each one. In 2005, a new measurement was done in the sample plots, when it was evaluated the changes in the horizontal structure, in the growth in diameter at $\mathrm{DBH}$ of all trees with circunference above $15 \mathrm{~cm}(\mathrm{CBH})$, the ingrowth and the mortality. In the Mazagão, alterations in relation to the number of species (52) and families (27) in the period from 2000 to 2005 had not occurred. The absolute density increased in 78 trees per hectare and the basal area increased $4,01 \mathrm{~m}^{2} / \mathrm{ha}$. In the Maniva river in 2000 they had 48 species of 24 families; in 2005 it appeared one new species and one family. The absolute density increased in 119 trees per hectare. The basal area increased in 4,96 m²/ha. In the Mutuacá River in 2000 they had 76 species of 32 families; in 2005 appeared five new species, two disappeared and the number of families kept the same. The basal area diminished in $3,05 \mathrm{~m}^{2} / \mathrm{ha}$.

Keywords: Fitossociology; Amazonian estuary; forestry dynamic.
\end{abstract}

\section{INTRODUÇ̃̃̃O}

O estado do Amapá, situado no extremo norte do Brasil, possui uma população em torno de 500 mil habitantes, sendo que mais de $80 \%$ vivem na zona urbana. Dos 100 mil que habitam a zona rural, mais de $50 \%$ sobrevivem dos recursos da várzea. Além dos amapaenses, os habitantes das ilhas do Pará, formadas por localidades do município de Afuá e de Gurupá, constituem um total populacional de mais de 50 mil pessoas que mantêm relações socioeconômicas com o estado do Amapá (QUEIROZ, 2004).

Rios e lagos da hiléia são muitas vezes acompanhados por faixas de terrenos baixos, sujeitos a inundação durante um determinado período de cada ano. Essas terras baixas são chamadas várzeas, e a 
floresta que as cobre chama-se mata de várzea, em contraste com a mata da terra-firme. As terras baixas da zona costeira da hiléia e do grande estuário amazônico são inundadas pela repercussão das marés atlânticas. A "mata" dos lugares mais baixos, diariamente inundados, aproxima-se do igapó, e a dos lugares mais altos, inundados somente pelas marés grandes, assemelha-se à da várzea de outras partes da região (DUCKE; BLACK, 1954).

Para Morán (1990), a várzea de estuário diferencia-se dos outros tipos de várzea da Amazônia pela influência diária da água salina, das marés e pela riqueza aquática. Para Hiraoka (1999), o estuário amazônico é caracterizado por terras baixas constituídas de sedimentos holocênicos, circundadas por depósitos mais antigos do Terciário, um pouco mais elevados, da Formação Barreiras. Para Rabelo (1999), os estuários são regiões mais amplas que as várzeas, sob influência diária das marés, na foz dos rios.

As várzeas são ambientes frágeis, com origem e funcionamento ligados à deposição de sedimentos geologicamente recentes, profundamente influenciados pelos regimes de marés e de águas pluviais. São as chamadas planícies de inundação, planícies quaternárias, planícies aluviais, etc. A essas mesmas condições deve-se a formação de solos com bons níveis de nutrientes e estoques biológicos ainda precariamente conhecidos. As utilizações desse ambiente estão centradas no extrativismo vegetal, principalmente açaí (fruto e palmito), seringa, andiroba, madeira e pecuária extensiva (AMAPÁ, 2000).

A floresta de várzea constitui o segundo maior ambiente florestado da região, considerando-se estrutura, diversidade e representatividade espacial. Em sua área de abrangência, sua maior concentração ocorre principalmente em margens de rios de água barrenta, onde, de certo modo, passa a ser regulada pelos regimes de marés. As maiores florestas de várzea do estado do Amapá ocorrem ao longo da orla amazônica, adentrando pelos estuários e baixos cursos dos inúmeros rios que aí deságuam (AMAPÁ, 2002).

A vida ribeirinha reproduz em suas generalidades as multi-relações estabelecidas pelas populações locais com o meio natural circundante. O rio, com seus regimes de cheias e vazantes, regula todo o fluxo de transporte, do suprimento alimentar e, em alguns casos, dos excedentes comercializáveis, dos padrões ou modelos de ocupação espacial, da edificação residencial, da natureza e desenvolvimento da floresta, da formação do solo, enfim, das próprias estratégias humanas em suas mais amplas dimensões de trabalho e de dependências sociais, culturais e econômicas (RABELO et al., 2005).

O padrão dominante de extrativismo na região de várzea do estuário amazônico, nos estados do Pará e do Amapá, envolve pouca preocupação com a sua sustentabilidade, razão pela qual os estoques de recursos, como madeira, peixes, camarão e outros de alto valor econômico, encontram-se sobreexplorados. Durante mais de meio século, a madeira foi o principal recurso econômico do estado e a indústria madeireira a principal fonte de emprego da população rural do Amapá e do vizinho estado do Pará (BARROS; UHL, 1995).

$\mathrm{O}$ açaizeiro é uma das plantas mais abundantes e freqüentes nas áreas de várzea, constituindo-se na espécie nativa de maior importância econômica para a região do estuário amazônico. Estudos mostram que a concentração de açaizeiros pode atingir até $25 \%$ da população botânica das áreas de várzea (ANDERSON et al., 1985).

Em estudos realizados em Igarapé Miri, em áreas com a população original pouco alterada, Nogueira (1999) encontrou populações de açaizeiros até cinco vezes maiores do que aquelas verificadas por outros estudiosos. Segundo o autor, isso decorreu da intensa exploração efetuada pelos habitantes do local estudado, os quais eliminaram quase que por completo as espécies consideradas de baixo valor comercial, de ocorrência natural nas áreas de várzea.

Em estudos realizados por Queiroz e Mochiutti (2002), sobre os tipos de açaizais do estuário amazônico e o efeito das intervenções praticadas por extratores ribeirinhos, os resultados mostraram que não se pode chamar de manejo às atuais intervenções praticadas e que os impactos são altos, observandose, em algumas áreas, a total supressão das palmeiras que concorrem com os açaizeiros.

Para aplicação de projetos corretos de Manejo Silvicultural, assim como o aproveitamento permanente de uma floresta, deve-se conhecer sua composição e a sua estrutura. Os resultados das análises estruturais permitem fazer deduções sobre a origem, características ecológicas e sinecológicas, dinamismo e tendências do futuro desenvolvimento das florestas, elementos básicos para o planejamento do manejo silvicultural (HOSOKAWA, 1986).

Em relação às espécies madeireiras e não-madeireiras que ocorrem no ambiente estuarino, Queiroz (2004), em estudo realizado no braço norte do rio Amazonas, no estado do Amapá, em 10 hectares distribuídos ao longo do grande rio e de seus tributários, encontrou um total de 116 espécies, 
com destaque para o açaizeiro (Euterpe oleracea Mart.), considerada a espécie mais importante do ponto de vista socioeconômico, e para as madeiráveis andiroba (Carapa guinensis Aubl.), anani (Symphonia globulifera L.), virola (Virola surinamensis Warb.), macacaúba (Platymiscium filipes Benth.), pracuúba (Mora paraensis Ducke) e pau-mulato (Callycophyllum spruceanum Benth.), entre outras.

De acordo com Falesi e Silva (1999), o tipo climático predominante em áreas de várzea do município de Santarém, de Alenquer e de Monte Alegre é o Ami da classificação de Köppen. Tipo climático semelhante foi encontrado por Vasquez e Rabelo (1999) nas áreas de várzea do Amapá. Para eles, a região estuarina amapaense se caracteriza por apresentar altas temperaturas (média anual de 27 ${ }^{\circ} \mathrm{C}$ ), alta umidade relativa (acima de $80 \%$ ), elevado índice pluviométrico (média anual variando entre 2000 e $2500 \mathrm{~mm}$ ), com um pequeno período seco de 3 a 4 meses e outro chuvoso (dezembro/junho).

Em estudos realizados na ilha de Santana, município de Santana, estado do Amapá, Valente et al. (1998) detectaram a ocorrência de dois tipos predominantes de solos, muito comuns na região do estuário, que representam muito bem os solos existentes nas áreas onde as parcelas amostrais foram instaladas: Gleissolo Háplico e Neossolo Flúvico.

O presente trabalho de pesquisa teve como objetivo principal analisar a estrutura e a dinâmica de floresta de várzea estuarina amazônica, no município de Mazagão, estado do Amapá, e área próxima, com a qual mantém intenso relacionamento socioeconômico.

\section{MATERIAL E MÉTODOS}

A localização das unidades amostrais foi feita em 2000, com a colaboração de membros da equipe realizadora do Zoneamento Ecológico Econômico do estado do Amapá, com base em informações oriundas da interpretação de imagens de satélite.

Foram instaladas duas amostras em várzea alta, sendo uma no rio Maniva, na região das ilhas do Pará, município de Afuá (PA), e outra no Furo do Mazagão, no município de Mazagão (AP), e uma amostra em várzea baixa, no rio Mutuacá, distrito de Carvão, município de Mazagão (Figura 1).

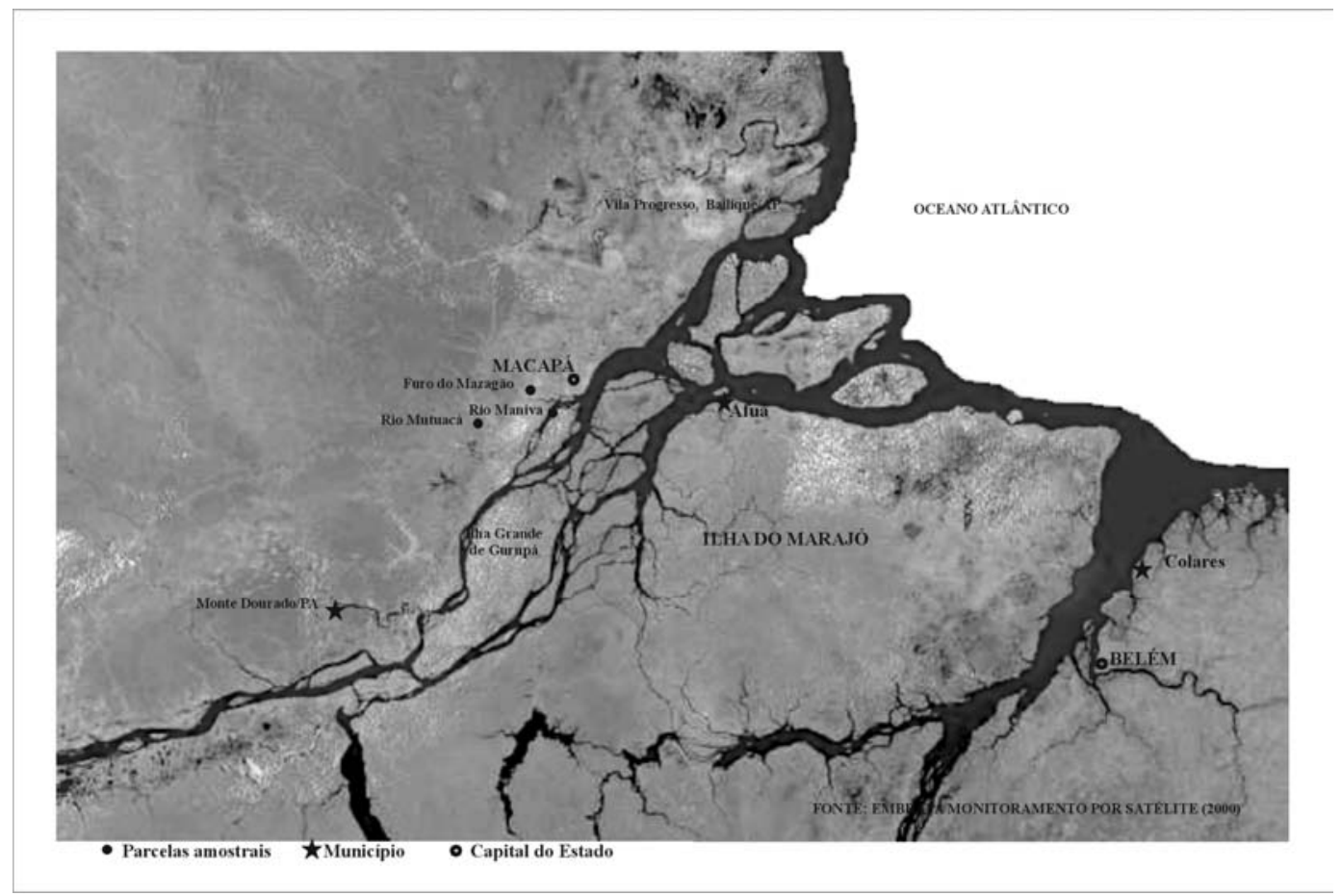

Figure 1. Localization of the study areas.

FLORESTA, Curitiba, PR, v. 37, n. 3, set./dez. 2007. 
Para a instalação das unidades amostrais, realizou-se levantamento de informações junto aos moradores locais sobre a ocorrência e localização de áreas de floresta de várzea, cujo estágio de desenvolvimento da vegetação fosse o de clímax, ou que a última extração de madeiras tivesse ocorrido de forma seletiva, com o mínimo de impacto e cuja última intervenção tivesse ocorrido a, pelo menos, 10 anos.

Para o estudo da vegetação foram considerados todos os indivíduos com CAP (Circunferência à Altura do Peito) maior ou igual a 15,0 cm. Para a composição florística, considerando que cada touceira de açaizeiros tem origem de apenas uma semente, portanto com estipes geneticamente iguais, contou-se cada touceira como apenas um indivíduo.

Foram instaladas três parcelas amostrais, sendo duas na várzea alta e uma na várzea baixa. Em cada local instalou-se uma parcela amostral de um hectare $(100$ x $100 \mathrm{~m})$, com dez subparcelas de 20 x 50 $\mathrm{m}$, de forma sistemática.

Para a realização dos estudos, foram coletados os seguintes dados: nome vulgar das espécies e a circunferência medida a 1,30 m de altura, a qual foi mensurada com auxílio de fita métrica de $1,50 \mathrm{~m}$. A altura foi obtida com auxílio de uma vara de 5,0 m. As alturas superiores e inferiores a 5,0 m foram obtidas por estimativa, tendo como referência a medida da vara.

A identificação das árvores foi feita por pessoas com experiência na identificação de árvores do ambiente estuarino, e as espécies sobre as quais se tinham dúvidas foram levadas para o herbário do estado do Amapá, para comparação com exsicatas dos referidos materiais.

A composição florística foi analisada através da comparação da distribuição dos indivíduos, agrupados em famílias botânicas e espécies.

Para a análise da estrutura horizontal, determinou-se a área basal por espécies e por famílias e os parâmetros fitossociológicos: freqüência absoluta, freqüência relativa, densidade absoluta, densidade relativa, dominância absoluta, dominância relativa, valor de importância das espécies e das famílias, quociente de mistura de Jentsch, índice de McGuinnes e índice de diversidade de Shannon $\left(\mathrm{H}^{\prime}\right.$ - $\left.\mathrm{J}^{\prime}\right)$.

$\mathrm{O}$ quociente de mistura de Jentsch foi obtido da relação entre o número de espécies e o número de indivíduos que ocorreram em uma mesma área, indicando a relação entre o número de indivíduos e a ocorrência de espécies, citado por Rabelo (1999).

A densidade absoluta foi calculada de acordo com o entendimento de Curtis e McIntosh (1950), que a consideram como o número total de indivíduos por unidade de área. A densidade relativa levou em consideração Mueller-Dombois e Ellenberg (1974), expressando, em porcentagem, a participação de cada espécie em relação ao número total de indivíduos de todas as espécies.

A freqüência absoluta de uma espécie é obtida pela porcentagem das parcelas em que a espécie ocorre, e a relativa expressa a relação entre freqüência absoluta de uma determinada espécie e a das demais, em porcentagem.

A dominância absoluta foi obtida pela soma das áreas transversais dos indivíduos de uma mesma espécie para cada área, e a relativa obteve-se da participação, em porcentagem, de cada espécie em relação à área basal total. Foi considerada a área transversal calculada a partir do diâmetro medido a 1,30 $\mathrm{m}$ do solo.

O valor de importância é obtido somando-se, para cada espécie, os valores relativos de densidade, freqüência e dominância, de acordo com Curtis (1959).

O índice de McGuinnes, utilizado para avaliar o grau de agregação das espécies, foi obtido da relação entre a densidade observada e a densidade esperada, de acordo com McGuinnes, citado por Barros e Machado (1984). De acordo com o valor encontrado, tem-se que: D/d > 1 indica uma tendência da espécie ao agrupamento; $\mathrm{D} / \mathrm{d}>2$ sugere que a espécie apresenta um padrão de distribuição contagiosa; $\mathrm{D} / \mathrm{d}=1$ indica que a espécie apresenta tendência de distribuição aleatória e $\mathrm{D} / \mathrm{d}<1$ sugere que a espécie tem uma distribuição uniforme.

O índice de diversidade de Shannon (H'), foi obtido da somatória do produto entre a abundância relativa de cada espécie e o logaritmo neperiano desse mesmo valor, sendo a somatória precedida do valor negativo, citado por Rabelo (1999).

A equabilidade ( $\mathrm{J}$ ) foi obtida da relação entre o índice de diversidade de Shannon e o logaritmo neperiano do número de espécies, citado por Rabelo (1999). 


\section{RESULTADOS E DISCUSSÃO}

Para a composição florística de um determinado ambiente, em dado momento, além da forma e da freqüência de utilização, deve-se considerar o tipo de utilização que tenha sido feito da vegetação (extração de madeiras, extração de palmito, instalação de roçados, entre outros), pois as ações antrópicas anteriores são fundamentais para as atuais expressões da composição florística e estruturais da área de floresta em estudo.

No levantamento realizado em 2005, foram encontradas 93 espécies, sendo 85 conhecidas e 8 não-identificadas, incluídas em 36 famílias, sendo 35 conhecidas e uma não-identificada (Tabela 1).

Tabela 1. Famílias e respectivas espécies com seus nomes científicos e comuns e ocorrência nas áreas estudadas, em 2005.

Table 1. Tree families and respective species with their scientific and common names and their occurrence in the studied areas in 2005.

\begin{tabular}{|c|c|c|c|c|c|}
\hline Família & Nome científico & Nome comum & Mut & Man & Maza \\
\hline \multirow[t]{2}{*}{ Anacardiaceae } & Spondias Mombim L. & Taperebá & $\mathrm{x}$ & - & - \\
\hline & Tapirira guianensis Aubl. & Tatapiririca & $\mathrm{x}$ & - & - \\
\hline Annonaceae & Guateria poeppigiana Mart. & Envira-preta & $\mathrm{x}$ & $\mathrm{x}$ & $\mathrm{x}$ \\
\hline \multirow[t]{2}{*}{ Apocynaceae } & Aspidosperma desmanthum Benth. & Jacamim & $\mathrm{x}$ & - & - \\
\hline & Aspidosperma auriculatum Benth. & Pau-de-arara & $\mathrm{x}$ & - & - \\
\hline \multirow[t]{4}{*}{ Bombacaceae } & Bombax munguba Mart. ex Zucc. & Munguba & $\mathrm{x}$ & - & - \\
\hline & Matisia paraensis Huber. & Cupuçurana & $\mathrm{x}$ & $\mathrm{x}$ & $\mathrm{x}$ \\
\hline & Quararibea guianensis Aubl. & Inajarana & $\mathrm{x}$ & $\mathrm{x}$ & $\mathrm{x}$ \\
\hline & Pachira aquatica Aubl. & Mamorana & $\mathrm{x}$ & - & - \\
\hline Burseraceae & Protium spruceanum Engl. & Breu-branco & $\mathrm{x}$ & - & $\mathrm{x}$ \\
\hline \multirow[t]{9}{*}{ Caesalpiniaceae } & Campsiandra laurifolia Benth. & Acapurana & $\mathrm{x}$ & $\mathrm{x}$ & $\mathrm{x}$ \\
\hline & Crudia oblonga Benth. & Ingá-vermelho/Ipé & $\mathrm{x}$ & $\mathrm{x}$ & $\mathrm{x}$ \\
\hline & Hymenaea oblongifolia Huber. & Jutaí-folha-fina & - & $\mathrm{x}$ & $\mathrm{x}$ \\
\hline & Macrolobium acaciaefolium Benth. & Arapari & - & - & $\mathrm{x}$ \\
\hline & Macrolobium augustifolium R.S.Cowan & Jutaí-folha-larga & $\mathrm{x}$ & $\mathrm{x}$ & - \\
\hline & Mora paraensis Ducke & Pracuúba & $\mathrm{x}$ & $\mathrm{x}$ & $\mathrm{x}$ \\
\hline & Swartzia cardiosperma Spr. Ex. Benth. & Pacapeuá & $\mathrm{x}$ & $\mathrm{x}$ & $\mathrm{x}$ \\
\hline & Tachigalia myrmecophila Ducke & Taxi-preto & $\mathrm{x}$ & - & - \\
\hline & Tachigalia paniculata Aubl. & Taxi-branco & $\mathrm{x}$ & - & - \\
\hline Caryocaraceae & Caryocar glabrum (Aubl.) Pers. & Piquiarana & $\mathrm{x}$ & $\mathrm{x}$ & $\mathrm{x}$ \\
\hline Cecropiaceae & Cecropia palmata Willd. & Embaúba & $\mathrm{x}$ & - & - \\
\hline \multirow[t]{4}{*}{ Chrysobalanaceae } & Licania heteromorpha Benth. & Macucu & $\mathrm{x}$ & $\mathrm{x}$ & $\mathrm{x}$ \\
\hline & Licania kunthiana H.F. & Cariperana & $\mathrm{x}$ & - & - \\
\hline & Licania macrophylla Benth. & Anoerá & $\mathrm{x}$ & $\mathrm{x}$ & $\mathrm{x}$ \\
\hline & Parinari excelsa Sabine & Isqueiro/Paranari & $\mathrm{x}$ & $\mathrm{x}$ & - \\
\hline \multirow[t]{5}{*}{ Clusiaceae } & Calophyllum brasiliensis Cambess. & Jacareúba & - & $\mathrm{x}$ & $\mathrm{x}$ \\
\hline & Caraipa grandiflora Mart. & Tamaquaré & - & $\mathrm{x}$ & $\mathrm{x}$ \\
\hline & Rheedia acuminata (Ruiz et Pav.) Pl. et Jr. & Bacurizinho & $\mathrm{x}$ & $\mathrm{x}$ & - \\
\hline & Rheedia macrophylla (Mart.) Pl. et Jr. & Bacuri-pari & - & $\mathrm{x}$ & - \\
\hline & Symphonia globulifera L.F. & Anani & $\mathrm{x}$ & $\mathrm{x}$ & $\mathrm{x}$ \\
\hline \multirow[t]{3}{*}{ Combretaceae } & Combretum cacoucia Excell \& Sandw. & Ioioca & $\mathrm{x}$ & $\mathrm{x}$ & - \\
\hline & Terminalia dichotoma $\mathrm{G}$. Meyer & Cuiarana & - & - & $\mathrm{x}$ \\
\hline & Terminalia guianensis Aubl. & Cinzeiro & $\mathrm{x}$ & - & - \\
\hline \multirow[t]{2}{*}{ Euphorbiaceae } & Hevea brasiliensis Muell. Arg. & Seringueira & $\mathrm{x}$ & $\mathrm{x}$ & $\mathrm{x}$ \\
\hline & Hura crepitans L. & Assacu & $\mathrm{x}$ & - & - \\
\hline \multirow[t]{6}{*}{ Fabaceae } & Crudia glaberrima (Steud.) Macbr. & Cumarurana & $\mathrm{x}$ & $\mathrm{x}$ & $\mathrm{x}$ \\
\hline & Ormosia coutinhoi Ducke & Buiuçu & $\mathrm{x}$ & - & - \\
\hline & Platymiscium filipes Benth. & Macacaúba & $\mathrm{x}$ & - & $\mathrm{x}$ \\
\hline & Pterocarpus amazonicus Huber. & Mututi & $\mathrm{x}$ & $\mathrm{x}$ & $\mathrm{x}$ \\
\hline & Pterocarpus officinalis Jacq. & Mututirana & $\mathrm{x}$ & - & $\mathrm{x}$ \\
\hline & Vatairea guianensis Aubl. & Faveira & $\mathrm{x}$ & $\mathrm{x}$ & $\mathrm{x}$ \\
\hline Flacourtiaceae & Banara guianensis Aubl. & Andorinheira & - & $\mathrm{x}$ & - \\
\hline Hernandiaceae & Hernandia guianensis Aubl. & Ventosa & $\mathrm{x}$ & $\mathrm{x}$ & - \\
\hline
\end{tabular}




\begin{tabular}{|c|c|c|c|c|c|}
\hline Família & Nome científico & Nome comum & Mut & Man & Maza \\
\hline Humiriaceae & Saccoglottis guianensis Aubl. & Uxirana & $\mathrm{x}$ & - & $\mathrm{x}$ \\
\hline Icacinaceae & Dendrobangia boliviana Rusby & Caferana & $\mathrm{x}$ & - & - \\
\hline \multirow[t]{3}{*}{ Lauraceae } & Aniba puchury-minor (Mart.) Mez. & Louro-amarelo & $\mathrm{x}$ & - & - \\
\hline & Licaria canella (Meiss.) Kosterm & Louro-pretinho & $\mathrm{x}$ & $\mathrm{x}$ & - \\
\hline & Licaria mahuba (Kuhlm. \& Samp.) Kosterm & Maúba & $\mathrm{x}$ & $\mathrm{x}$ & $\mathrm{x}$ \\
\hline Lauraceae & Ocotea $\mathrm{sp}$ & Louro-branco & $\mathrm{x}$ & - & - \\
\hline \multirow[t]{2}{*}{ Lecythidaceae } & Alantoma lineata Miers & Ceru & $\mathrm{x}$ & - & - \\
\hline & Gustavia augusta $\mathrm{L}$. & Jenipaparana & - & - & $\mathrm{x}$ \\
\hline Malpiguiaceae & Spdesc & Canela-de-velho & $\mathrm{x}$ & - & - \\
\hline \multirow[t]{2}{*}{ Melastomataceae } & Miconia ceramicarpa Cogn. & Papa-terra & $\mathrm{x}$ & - & $\mathrm{x}$ \\
\hline & Mouriri acutiflora Naud. & Camutim & $\mathrm{x}$ & - & $\mathrm{x}$ \\
\hline \multirow{4}{*}{ Meliaceae } & Carapa guianensis Aubl. & Andiroba & $\mathrm{x}$ & $\mathrm{x}$ & $\mathrm{x}$ \\
\hline & Cedrela odorata $\mathrm{L}$. & Cedro & $\mathrm{x}$ & - & - \\
\hline & Trichilia paraensis C. DC. & Jataúba & $\mathrm{x}$ & - & - \\
\hline & Trichilia surinamensis (Miq.) C. DC. & Marajoão & $\mathrm{x}$ & - & - \\
\hline \multirow[t]{8}{*}{ Mimosaceae } & Inga cinnamomea Spruce Ex. Benth. & Ingá-branco & $\mathrm{x}$ & $\mathrm{x}$ & $\mathrm{x}$ \\
\hline & Inga sp1 & Ingarana & $\mathrm{x}$ & - & - \\
\hline & Inga $\mathrm{sp} 2$ & Ingá & $\mathrm{x}$ & - & - \\
\hline & Inga $\mathrm{sp} 3$ & Ingá-de-velho & $\mathrm{x}$ & - & - \\
\hline & Pentaclethra macroloba (Willd.) O. Kuntze & Pracaxi & $\mathrm{x}$ & $\mathrm{x}$ & $\mathrm{x}$ \\
\hline & Pithecellobium inaequale (H.B.K.) Benth. & Jaranduba-da-mata & $\mathrm{x}$ & $\mathrm{x}$ & $\mathrm{x}$ \\
\hline & Pithecellobium sp & Jaranduba & $\mathrm{x}$ & - & $\mathrm{x}$ \\
\hline & Swartzia acuminata Willd. & Pitaíca & - & $\mathrm{x}$ & $\mathrm{x}$ \\
\hline \multirow[t]{3}{*}{ Moraceae } & Ficus maxima (P.) Miller & Caxinguba & $\mathrm{x}$ & - & - \\
\hline & Ficus pertusa C.F. & Apuí & $\mathrm{x}$ & - & - \\
\hline & Olmedia caloneura Huber. & Muiratinga & $\mathrm{x}$ & $\mathrm{x}$ & $\mathrm{x}$ \\
\hline Myristicaceae & Virola surinamensis (Rol.) Warb. & Virola & $\mathrm{x}$ & $\mathrm{x}$ & $\mathrm{x}$ \\
\hline Myrsinaceae & Ardisia sp & Olho-de-galega & - & $\mathrm{x}$ & $\mathrm{x}$ \\
\hline \multirow[t]{2}{*}{ Myrtaceae } & Calyptranthes speciosa Sagot. & Goiabarana & $\mathrm{x}$ & $\mathrm{x}$ & $\mathrm{x}$ \\
\hline & Eugenia browsnbergii Amshoff & Goiaba-braba & $\mathrm{x}$ & $\mathrm{x}$ & $\mathrm{x}$ \\
\hline Olacaceae & Heisteria acuminata (HPB) Engl. & Açaí-pretinho & $\mathrm{x}$ & $\mathrm{x}$ & $\mathrm{x}$ \\
\hline Rubiaceae & Callycophyllum spruceanum Benth. & Pau-mulato & $\mathrm{x}$ & $\mathrm{x}$ & $\mathrm{x}$ \\
\hline Rutaceae & Metrodorea flavida Krause. & Laranjinha & $\mathrm{x}$ & $\mathrm{x}$ & - \\
\hline \multirow[t]{3}{*}{ Sapotaceae } & Crysophyllum excelsum Huber. & Guajaraí & $\mathrm{x}$ & - & - \\
\hline & Pouteria bilocularis (Winkler) Baehni & Abiurana & - & - & $\mathrm{x}$ \\
\hline & Pouteria sagotiana (Baill) Eyma. & Maçaranduba & $\mathrm{x}$ & $\mathrm{x}$ & $\mathrm{x}$ \\
\hline \multirow{4}{*}{ Sterculiaceae } & Guazuma ulmifolia Lam. & Mutamba & $\mathrm{x}$ & - & - \\
\hline & Herrania mariae (Mart.) Dene. & Cacau-jacaré & $\mathrm{x}$ & - & - \\
\hline & Sterculia speciosa K. Schum & Capoteiro & $\mathrm{x}$ & $\mathrm{x}$ & $\mathrm{x}$ \\
\hline & Theobroma Cacao L. & Cacau & $\mathrm{x}$ & - & $\mathrm{x}$ \\
\hline Tiliaceae & Apeiba burchelii Sprague. & Chapéu-de-sol & $\mathrm{x}$ & $\mathrm{x}$ & $\mathrm{x}$ \\
\hline Famdesc & Spdesc & Avineira & - & - & $\mathrm{x}$ \\
\hline \multirow[t]{8}{*}{ Arecaceae } & Astrocaryum mumbaca Mart. & Mumbaca & $\mathrm{x}$ & - & $\mathrm{x}$ \\
\hline & Astrocaryum murumuru Mart. & Murumuru & $\mathrm{x}$ & $\mathrm{x}$ & $\mathrm{x}$ \\
\hline & Attalea excelsa Mart. & Urucuri & $\mathrm{x}$ & $\mathrm{x}$ & - \\
\hline & Manicaria saccifera Gaertn. & Bussu & $\mathrm{x}$ & $\mathrm{x}$ & $\mathrm{x}$ \\
\hline & Mauritia flexuosa $\mathrm{L}$. & Buriti & - & $\mathrm{x}$ & $\mathrm{x}$ \\
\hline & Oenocarpus bacaba Mart. & Bacaba & $\mathrm{x}$ & - & - \\
\hline & Socratea exhorriza (Mart.) Wendl. & Paxiúba & - & $\mathrm{x}$ & - \\
\hline & Euterpe oleraceae Mart. & Açaí & $\mathrm{x}$ & $\mathrm{x}$ & $\mathrm{x}$ \\
\hline Total & & & 79 & 49 & 52 \\
\hline
\end{tabular}

Mut: rio Mutuacá; Man: rio Maniva; Maza: Furo do Mazagão; x: presença da espécie.

No Furo Mazagão, o proprietário da área estudada há muito vive da coleta de frutos de açá e da comercialização da polpa fresca. As intervenções no ambiente natural têm a função principal de limpar o local para facilitar a caminhada na floresta e o acesso aos cachos de açaí para coleta dos frutos. Nos últimos dez anos, priorizou as atividades no açaizal, coletando os frutos e extraindo e comercializando a polpa, além de vender o excedente de frutos no mercado de Mazagão. A extração de madeira ocorre 
quando da necessidade de construção de casas ou barracões na própria área e é feita priorizando o desenvolvimento dos açaizeiros. Observa-se que o açaizal foi moderadamente ampliado na propriedade, com impacto mínimo na população das dicotiledôneas.

No Furo do Mazagão não ocorreram alterações em relação ao número de espécies (52) e de famílias (27) no período de 2000 a 2005. O quociente de mistura de Jentsch passou de 01:16 para 01:18, relação bem representativa para a floresta de várzea estuarina que está em clímax. Quanto ao índice de McGuinnes (IGA), 5 espécies (9,6\%) mudaram o padrão de comportamento. O índice de diversidade de Shannon $\left(H^{\prime}\right)$ passou de 2,68 para 2,70 , e a equabilidade se manteve inalterada $(0,68)$. A densidade aumentou de 872 para 950 árvores por hectare, um incremento de $8,9 \%$. A área basal cresceu de 31,82 para $35,83 \mathrm{~m}^{2} /$ ha, um incremento de $12,6 \%$. As espécies com ingressos mais significativos foram Mora paraensis, com 39 indivíduos (31,0\%); Licania macrophylla, com 7 (13,0\%); Pithecellobium inaequale, com $6(28,6 \%)$; Pentaclethra macroloba, com $6(10,7 \%)$ e Astrocaryum murumuru, com $6(4,4 \%)$ (Tabela 2).

Tabela 2. Valores absolutos de freqüência, densidade, dominância e índice de McGuinnes (IGA) para as espécies no Furo do Mazagão.

Table 2. Absolute values of frequency, density, dominance and McGuinnes (IGA) index for the species in the Mazagão.

\begin{tabular}{|c|c|c|c|c|c|c|c|c|c|c|c|c|}
\hline \multirow{2}{*}{ Espécies } & \multicolumn{6}{|c|}{ Agosto de 2000} & \multicolumn{6}{|c|}{ Agosto de 2005} \\
\hline & FA & DA & DoA & VI & IGA & COMP. & FA & DA & DoA & VI & IGA & COMP. \\
\hline Euterpe oleracea & 100 & 222 & 5,87 & 48,87 & 4,8 & Cont. & 100 & 223 & 6,13 & 45,40 & 4,8 & Cont. \\
\hline Mora paraensis & 100 & 126 & 6,94 & 41,21 & 2,7 & Cont. & 100 & 161 & 8,09 & 44,36 & 3,5 & Cont. \\
\hline Astrocaryum murumuru & 100 & 137 & 2,06 & 27,13 & 3,0 & Cont. & 100 & 141 & 2,42 & 26,42 & 3,1 & Cont. \\
\hline Pentaclethra macroloba & 100 & 56 & 1,51 & 16,12 & 1,2 & Agrup. & 100 & 60 & 1,73 & 15,96 & 1,3 & Agrup. \\
\hline Licania macrophylla & 70 & 54 & 1,78 & 15,27 & 4,5 & Cont. & 90 & 60 & 1,99 & 16,22 & 2,6 & Cont. \\
\hline Carapa guianensis & 90 & 27 & 1,32 & 11,71 & 1,2 & Agrup. & 90 & 28 & 1,54 & 11,61 & 1,2 & Agrup. \\
\hline Licania heteromorpha & 60 & 19 & 1,91 & 11,17 & 2,1 & Cont. & 60 & 21 & 2,19 & 11,22 & 2,3 & Cont. \\
\hline Macrolobium microcarpum & 30 & 5 & 1,99 & 8,31 & 1,4 & Agrup. & 30 & 5 & 2,22 & 8,17 & 1,4 & Agrup. \\
\hline Miconia ceramicarpa & 80 & 28 & 0,30 & 8,13 & 1,7 & Agrup. & 80 & 31 & 0,35 & 8,12 & 1,9 & Agrup. \\
\hline Matisia paraensis & 90 & 15 & 0,57 & 7,97 & 0,7 & Unif. & 90 & 15 & 0,67 & 7,80 & 0,7 & Unif. \\
\hline Pithecellobium inaequale & 100 & 21 & 0,12 & 7,72 & 0,5 & Unif. & 100 & 26 & 0,16 & 8,01 & 0,6 & Unif. \\
\hline Virola surinamenis & 70 & 16 & 0,77 & 7,73 & 1,3 & Agrup. & 80 & 18 & 0,90 & 8,26 & 1,1 & Agrup. \\
\hline Quararibea guianensis & 90 & 16 & 0,39 & 7,52 & 0,7 & Unif. & 90 & 18 & 0,46 & 7,53 & 0,8 & Unif. \\
\hline Pterocarpus amazonicus & 50 & 16 & 0,87 & 7,05 & 2,3 & Cont. & 40 & 17 & 0,69 & 5,66 & 3,3 & Cont. \\
\hline Manicaria saccifera & 40 & 10 & 0,71 & 5,36 & 2,0 & Agrup. & 40 & 10 & 0,83 & 5,29 & 2,0 & Agrup. \\
\hline Guatteria poeppigiana & 40 & 6 & 0,09 & 2,95 & 1,2 & Agrup. & 40 & 6 & 0,11 & 2,88 & 1,2 & Agrup. \\
\hline Protium pubescens & 10 & 1 & 0,00 & 0,62 & 0,9 & Unif. & 10 & 1 & 0,00 & 0,60 & 0,9 & Unif. \\
\hline Campsiandra laurifolia & 20 & 2 & 0,27 & 2,06 & 0,9 & Unif. & 20 & 5 & 0,31 & 2,36 & 2,2 & Cont. \\
\hline Crudia oblonga & 20 & 2 & 0,11 & 1,55 & 0,9 & Unif. & 30 & 4 & 0,22 & 2,48 & 1,1 & Agrup. \\
\hline Hymenaea oblongifolia & 10 & 1 & 0,02 & 0,68 & 0,9 & Unif. & 10 & 1 & 0,03 & 0,66 & 0,9 & Unif. \\
\hline Swartzia cardiosperma & 60 & 7 & 0,32 & 4,76 & 0,8 & Unif. & 60 & 8 & 0,36 & 4,75 & 0,9 & Unif. \\
\hline Caryocar grabrum & 20 & 2 & 0,02 & 1,29 & 0,9 & Unif. & 20 & 2 & 0,03 & 1,26 & 0,9 & Unif. \\
\hline Calophyllum brasiliensis & 30 & 3 & 0,14 & 2,27 & 0,8 & Unif. & 30 & 3 & 0,17 & 2,25 & 0,8 & Unif. \\
\hline Caraipa grandiflora & 30 & 3 & 0,03 & 1,93 & 0,8 & Unif. & 30 & 3 & 0,03 & 1,86 & 0,8 & Unif. \\
\hline Symphonia globulifera & 20 & 3 & 0,25 & 2,11 & 1,3 & Agrup. & 30 & 4 & 0,27 & 2,63 & 1,1 & Agrup. \\
\hline Terminalia dichotoma & 10 & 1 & 0,00 & 0,62 & 0,9 & Unif. & 10 & 1 & 0,00 & 0,60 & 0,9 & Unif. \\
\hline Hevea brasiliensis & 10 & 1 & 0,00 & 0,62 & 0,9 & Unif. & 10 & 1 & 0,00 & 0,60 & 0,9 & Unif. \\
\hline Dipteryx sp & 60 & 8 & 0,45 & 5,29 & 0,9 & Unif. & 60 & 8 & 0,51 & 5,17 & 0,9 & Unif. \\
\hline Platymiscium filipes & 10 & 1 & 0,07 & 0,85 & 0,9 & Unif. & 10 & 1 & 0,09 & 0,84 & 0,9 & Unif. \\
\hline Pterocarpus officinalis & 40 & 7 & 0,72 & 5,05 & 1,4 & Agrup. & 40 & 6 & 0,83 & 4,88 & 1,2 & Agrup. \\
\hline Vatairea guianensis & 20 & 2 & 0,36 & 2,36 & 0,9 & Unif. & 20 & 2 & 0,47 & 2,48 & 0,9 & Unif. \\
\hline Spdesc & 30 & 2 & 0,01 & 1,73 & 0,6 & Unif. & 20 & 2 & 0,01 & 1,20 & 0,9 & Unif. \\
\hline Saccoglottis guianensis & 20 & 3 & 0,04 & 1,45 & 1,3 & Agrup. & 20 & 3 & 0,05 & 1,43 & 1,3 & Agrup. \\
\hline Licaria mahuba & 30 & 6 & 0,36 & 3,32 & 1,7 & Agrup. & 30 & 6 & 0,40 & 3,19 & 1,7 & Agrup. \\
\hline
\end{tabular}




\begin{tabular}{|c|c|c|c|c|c|c|c|c|c|c|c|c|}
\hline \multirow{2}{*}{ Espécies } & \multicolumn{6}{|c|}{ Agosto de 2000} & \multicolumn{6}{|c|}{ Agosto de 2005} \\
\hline & FA & DA & DoA & VI & IGA & COMP. & FA & DA & DoA & VI & IGA & COMP. \\
\hline Gustavia augusta & 10 & 1 & 0,01 & 0,63 & 0,9 & Unif. & 10 & 1 & 0,01 & 0,61 & 0,9 & Unif. \\
\hline Mouriri acutiflora & 10 & 1 & 0,04 & 0,74 & 0,9 & Unif. & 10 & 2 & 0,05 & 0,83 & 1,9 & Agrup. \\
\hline Inga cinnamomea & 40 & 6 & 0,02 & 2,73 & 1,2 & Agrup. & 50 & 9 & 0,03 & 3,45 & 1,3 & Agrup. \\
\hline Pithecellobium sp & 30 & 4 & 0,10 & 2,25 & 1,1 & Agrup. & 20 & 2 & 0,01 & 1,21 & 0,9 & Unif. \\
\hline Swartzia platygyne & 10 & 1 & 0,01 & 0,63 & 0,9 & Unif. & 10 & 1 & 0,01 & 0,62 & 0,9 & Unif. \\
\hline Olmedia caloneura & 40 & 5 & 0,58 & 4,37 & 1,0 & Aleat. & 40 & 5 & 0,65 & 4,28 & 1,0 & Aleat. \\
\hline Ardisia sp & 10 & 1 & 0,00 & 0,62 & 0,9 & Unif. & 20 & 2 & 0,00 & 1,19 & 0,9 & Unif. \\
\hline Calyptranthes speciosa & 40 & 4 & 0,01 & 2,47 & 0,8 & Unif. & 50 & 6 & 0,02 & 3,10 & 0,9 & Unif. \\
\hline Eugenia browsbergii & 30 & 5 & 0,02 & 2,12 & 1,4 & Agrup. & 30 & 5 & 0,03 & 2,05 & 1,4 & Agrup. \\
\hline Heisteria acuminata & 20 & 2 & 0,01 & 1,24 & 0,9 & Unif. & 20 & 2 & 0,01 & 1,19 & 0,9 & Unif. \\
\hline Callycophyllum spruceanum & 10 & 1 & 0,17 & 1,13 & 0,9 & Unif. & 10 & 1 & 0,18 & 1,09 & 0,9 & Unif. \\
\hline Pouteria bilocularis & 30 & 3 & 0,24 & 2,60 & 0,8 & Unif. & 30 & 4 & 0,27 & 2,62 & 1,1 & Agrup. \\
\hline Pouteria sagotiana & 20 & 2 & 0,12 & 1,58 & 0,9 & Unif. & 20 & 2 & 0,15 & 1,60 & 0,9 & Unif. \\
\hline Sterculia speciosa & 20 & 3 & 0,04 & 1,46 & 1,3 & Agrup. & 20 & 3 & 0,05 & 1,41 & 1,3 & Agrup. \\
\hline Theobroma cacau & 10 & 1 & 0,00 & 0,62 & 0,9 & Unif. & 10 & 1 & 0,01 & 0,62 & 0,9 & Unif. \\
\hline Apeiba burchelii & 10 & 1 & 0,00 & 0,62 & 0,9 & Unif. & 10 & 1 & 0,00 & 0,60 & 0,9 & Unif. \\
\hline Astrocaryum mumbaca & 10 & 1 & 0,00 & 0,62 & 0,9 & Unif. & 10 & 1 & 0,00 & 0,59 & 0,9 & Unif. \\
\hline Mauritia flexuosa & 10 & 1 & 0,06 & 0,80 & 0,9 & Unif. & 10 & 1 & 0,07 & 0,78 & 0,9 & Unif. \\
\hline Total & 2020 & 872 & 31,8 & 300 & & & 2070 & 950 & 35,8 & 300 & & \\
\hline
\end{tabular}

FA: Freqüência absoluta; DA: Densidade absoluta; DoA: Dominância absoluta; VI: Valor de Importância; IGA: Índice de Mc Guinnes; COMP.: Comportamento; Cont.: Espécies com distribuição contagiosa; Agrup.: Espécies com tendência ao agrupamento; Unif.: Espécies com distribuição uniforme; Aleat.: Espécies com tendência de distribuição aleatória.

No rio Maniva, o proprietário da área manteve, até o final da década de 80, uma pequena serraria e fazia extração seletiva de madeira. Além disso, extraía palmito, em regime de baixo impacto, nas limpezas do açaizal, para abastecimento de fábricas de palmito instaladas nas proximidades da área. A partir do início da década de 90, dedicou-se à coleta de frutos de açaí para comercialização em Macapá e à extração seletiva da madeira apenas para usos pela própria família, para construção de casas e confecção de móveis e embarcações. $\mathrm{O}$ açaizal foi moderadamente ampliado, com impacto mínimo na população e diversidade das dicotiledôneas.

No rio Maniva, em 2000, havia 48 espécies de 24 famílias; em 2005 surgiu uma espécie e uma família novas, a pioneira Heisteria acuminata (HPB) Engl. (açaí-pretinho), da família Olacaceae, espécie do estrato inferior. O quociente de mistura de Jentsch passou de 01:16 para 01:18, como citado anteriormente, relação bem representativa para a floresta de várzea estuarina que está em clímax. Quanto ao índice de McGuinnes (IGA), 9 espécies (18,4\%) mudaram o padrão de comportamento. O índice de diversidade de Shannon ( $\left.\mathrm{H}^{\prime}\right)$ passou de 2,88 para 2,89 e a equabilidade se manteve inalterada $(0,74)$. A densidade aumentou de 807 para 926 árvores por hectare, um incremento de 14,7\%. A área basal cresceu de 33,68 para $38,64 \mathrm{~m}^{2} /$ ha, um incremento de $14,7 \%$. As espécies com ingressos mais significativos foram Euterpe oleracea, com 58 indivíduos (42,3\%); Quararibea guianensis, com 13 (40,6\%); Inga sp, com 9 (100\%); Pithecellobium inaequale, com 6 (35,3\%); e Carapa guianensis, com 5 (22,7\%) (Tabela $3)$.

No rio Mutuacá, o proprietário da área estudada sempre que precisa de dinheiro recorre aos recursos da área de floresta para obtê-lo, já que esta é sua principal fonte de renda. Já faz alguns anos que mudou para a área urbana, visitando a propriedade florestal com certa freqüência. Em 2003, visando atender demandas urgentes da família, realizou retirada seletiva de palmito e de madeira. Esse tipo de procedimento continua muito freqüente entre os extrativistas ribeirinhos, sendo que as alterações na estrutura da floresta resultam, muitas vezes, das pressões econômicas que a família enfrenta.

No rio Mutuacá, em 2000, havia 76 espécies de 32 famílias; em 2005 surgiram cinco espécies novas e o número de famílias se manteve o mesmo. As novas espécies, a maioria com comportamento de pioneiras são: Tachigalia myrmecophila Ducke (taxi-preto), Caesalpiniaceae; Ocotea sp (louro-branco), Lauraceae; Mouriri acutiflora Naud. (camutim), Melastomataceae; Pithecellobium inaequale (HBK) Benth. (jaranduba-da-mata), Mimosaceae; e Astrocaryum mumbaca Mart. (mumbaca), Arecaceae. Em 
2005 foram encontradas 79 espécies, com um aumento de 3 espécies. Duas espécies que ocorriam em 2000 não apareceram mais em 2005: Hymenaea oblongifolia Huber (jutaí-folha-fina), Caesalpiniaceae, e Vismia macrophylla H.B.K. (lacre), Clusiaceae. O quociente de mistura de Jentsch passou de 01:12 para 01:11. Quanto ao índice de McGuinnes (IGA), 12 espécies (15,8\%) mudaram o padrão de comportamento. O índice de diversidade de Shannon (H') passou de 2,98 para 3,08 e a equabilidade passou de 0,68 para 0,70. A densidade diminuiu de 961 para 903 árvores por hectare, uma redução de $6,0 \%$. A área basal diminuiu de 35,72 para $32,67 \mathrm{~m}^{2} /$ ha, uma redução de $8,5 \%$. Os resultados refletem as intervenções implementadas pelo proprietário na área de estudo (Tabela 4). As espécies com ingressos mais significativos foram: Pentaclethra macroloba, 9 (10,7\%); Pterocarpus amazonicus, 7 (38,9\%); Swartzia cardiosperma, 5 (38,5\%); Miconia ceramicarpa, 5 (12,2\%); e Cecropia palmata, 4 (14,3\%).

Tabela 3. Valores absolutos de freqüência, densidade, dominância e índice de McGuinnes (IGA) para as espécies no rio Maniva.

Table 3. Absolute values of frequence, density, dominance and McGuinnes (IGA) index for the species in the Maniva river.

\begin{tabular}{|c|c|c|c|c|c|c|c|c|c|c|c|c|}
\hline \multirow{2}{*}{ Espécies } & \multicolumn{5}{|c|}{ Novembro de 2000} & & \multicolumn{5}{|c|}{ Novembro de 2005} & \multirow[b]{2}{*}{ COMP. } \\
\hline & FA & DA & DoA & VI & IGA & COMP. & FA & DA & DoA & VI & IGA & \\
\hline Euterpe oleracea & 100 & 137 & 6,00 & 39,28 & 3,0 & Cont. & 100 & 183 & 6,31 & 40,39 & 4,0 & Cont. \\
\hline Astrocaryum murumuru & 100 & 141 & 1,98 & 27,83 & 3,1 & Cont. & 100 & 141 & 2,31 & 25,50 & 3,1 & Cont. \\
\hline Mora paraensis & 100 & 80 & 3,55 & 24,95 & 1,7 & Agrup. & 100 & 94 & 4,34 & 25,68 & 2,0 & Cont. \\
\hline Pentaclethra macroloba & 100 & 73 & 1,89 & 19,14 & 1,6 & Agrup. & 100 & 74 & 2,13 & 17,78 & 1,6 & Agrup. \\
\hline Manicaria saccifera & 90 & 55 & 2,65 & 18,71 & 2,4 & Cont. & 100 & 55 & 3,13 & 18,33 & 1,2 & Agrup. \\
\hline Matisia paraensis & 100 & 39 & 2,00 & 15,24 & 0,8 & Unif. & 100 & 39 & 2,22 & 14,25 & 0,8 & Unif. \\
\hline Swartzia acuminata & 90 & 10 & 2,38 & 12,34 & 0,4 & Unif. & 90 & 11 & 2,99 & 12,80 & 0,5 & Unif. \\
\hline Licania macrophylla & 90 & 27 & 1,56 & 12,02 & 1,2 & Agrup. & 90 & 29 & 1,49 & 10,85 & 1,3 & Agrup. \\
\hline Carapa guianensis & 100 & 22 & 1,28 & 11,01 & 0,5 & Unif. & 100 & 27 & 1,51 & 11,12 & 0,6 & Unif. \\
\hline Symphonia globulifera & 100 & 22 & 1,23 & 10,87 & 0,5 & Unif. & 100 & 23 & 1,47 & 10,58 & 0,5 & Unif. \\
\hline Quararibea guianensis & 100 & 32 & 0,52 & 10,01 & 0,7 & Unif. & 100 & 44 & 0,60 & 10,59 & 1,0 & Unif. \\
\hline Virola surinamensis & 80 & 13 & 0,95 & 8,02 & 0,8 & Unif. & 80 & 14 & 1,19 & 8,02 & 0,9 & Unif. \\
\hline Pterocarpus amazonicus & 80 & 18 & 0,58 & 7,54 & 1,1 & Agrup. & 90 & 22 & 0,68 & 7,99 & 1,0 & Aleat. \\
\hline Pithecellobium inaequale & 80 & 17 & 0,15 & 6,13 & 1,1 & Agrup. & 80 & 23 & 0,15 & 6,31 & 1,4 & Agrup. \\
\hline Swartzia cardiosperma & 40 & 10 & 0,75 & 5,26 & 2,0 & Agrup. & 50 & 14 & 0,83 & 5,81 & 2,0 & Cont. \\
\hline Guateria poeppigiana & 30 & 3 & 0,03 & 1,81 & 0,8 & Unif. & 30 & 3 & 0,04 & 1,71 & 0,8 & Unif. \\
\hline Campsiandra laurifolia & 10 & 1 & 0,01 & 0,59 & 0,9 & Unif. & 10 & 1 & 0,01 & 0,56 & 0,9 & Unif. \\
\hline Crudia oblonga & 10 & 1 & 0,01 & 0,60 & 0,9 & Unif. & 10 & 1 & 0,02 & 0,58 & 0,9 & Unif. \\
\hline Hymenaea oblongifolia & 20 & 2 & 0,02 & 1,22 & 0,9 & Unif. & 30 & 3 & 0,20 & 2,12 & 0,8 & Unif. \\
\hline Macrolobium augustifolium & 30 & 3 & 0,18 & & 0,8 & Unif. & 20 & 2 & 0,02 & 1,13 & 0,9 & Unif. \\
\hline Caryocar microcarpum & 30 & 3 & 0,23 & 2,40 & 0,8 & Unif. & 30 & 3 & 0,26 & 2,28 & 0,8 & Unif. \\
\hline Licania heteromorpha & 40 & 5 & 0,94 & 5,21 & 1,0 & Aleat. & 40 & 5 & 1,02 & 4,89 & 1,0 & Aleat. \\
\hline Parinarium excelsa & 10 & 1 & 0,02 & 0,63 & 0,9 & Unif. & 10 & 1 & 0,02 & 0,60 & 0,9 & Unif. \\
\hline Calophyllum brasiliensis & 10 & 1 & 0,00 & 0,59 & 0,9 & Unif. & 10 & 2 & 0,01 & 0,67 & 1,9 & Agrup. \\
\hline Caraipa grandiflora & 60 & 14 & 0,18 & 4,95 & 1,5 & Agrup. & 60 & 15 & 0,23 & 4,78 & 1,6 & Agrup. \\
\hline Rheedia acuminata & 20 & 2 & 0,00 & 1,16 & 0,9 & Unif. & 30 & 3 & 0,01 & 1,63 & 0,8 & Unif. \\
\hline Rheedia macrophylla & 20 & 2 & 0,01 & 1,18 & 0,9 & Unif. & 20 & 2 & 0,02 & 1,12 & 0,9 & Unif. \\
\hline Combretum cacoucia & 10 & 1 & 0,01 & 0,60 & 0,9 & Unif. & 40 & 4 & 0,01 & 2,19 & 0,8 & Unif. \\
\hline Hevea brasiliensis & 50 & 7 & 0,52 & 4,65 & 1,0 & Aleat. & 50 & 7 & 0,67 & 4,64 & 1,0 & Aleat. \\
\hline Dipteryx sp & 30 & 3 & 0,24 & 2,44 & 0,8 & Unif. & 30 & 3 & 0,27 & 2,32 & 0,8 & Unif. \\
\hline Vatairea guianensis & 30 & 4 & 0,43 & 3,11 & 1,1 & Agrup. & 30 & 5 & 0,56 & 3,28 & 1,4 & Agrup. \\
\hline Banara guianensis & 10 & 1 & 0,01 & 0,60 & 0,9 & Unif. & 10 & 1 & 0,01 & 0,56 & 0,9 & Unif. \\
\hline Hernandia guianensis & 50 & 7 & 0,15 & 3,56 & 1,0 & Aleat. & 60 & 8 & 0,18 & 3,91 & 0,9 & Unif. \\
\hline Licania canella & 10 & 1 & 0,08 & 0,81 & 0,9 & Unif. & 10 & 1 & 0,11 & 0,81 & 0,9 & Unif. \\
\hline Licaria mahuba & 60 & 7 & 0,25 & 4,31 & 0,8 & Unif. & 60 & 7 & 0,32 & 4,17 & 0,8 & Unif. \\
\hline Inga cinnamomea & 60 & 9 & 0,13 & 4,18 & 1,0 & Aleat. & 60 & 18 & 0,16 & 4,94 & 2,0 & Agrup. \\
\hline
\end{tabular}




\begin{tabular}{|c|c|c|c|c|c|c|c|c|c|c|c|c|}
\hline \multirow{2}{*}{ Espécies } & \multicolumn{6}{|c|}{ Novembro de 2000} & \multicolumn{6}{|c|}{ Novembro de 2005} \\
\hline & FA & DA & DoA & VI & IGA & COMP. & FA & DA & DoA & VI & IGA & COMP. \\
\hline Olmedia caloneura & 20 & 2 & 0,28 & 1,96 & 0,9 & Unif. & 20 & 2 & 0,33 & 1,93 & 0,9 & Unif. \\
\hline Ardisia sp & 20 & 2 & 0,00 & 1,16 & 0,9 & Unif. & 20 & 2 & 0,00 & 1,09 & 0,9 & Unif. \\
\hline Calyptranthes speciosa & 20 & 2 & 0,01 & 1,17 & 0,9 & Unif. & 20 & 3 & 0,01 & 1,22 & 1,3 & Agrup. \\
\hline Eugenia brawsbergii & 20 & 4 & 0,03 & 1,48 & 1,8 & Agrup. & 20 & 6 & 0,04 & 1,61 & 2,7 & Cont. \\
\hline Callycophyllum spruceanum & 20 & 2 & 0,75 & 3,36 & 0,9 & Unif. & 20 & 2 & 0,81 & 3,17 & 0,9 & Unif. \\
\hline Metrodorea flavida & 10 & 1 & 0,00 & 0,58 & 0,9 & Unif. & 20 & 2 & 0,01 & 1,09 & 0,9 & Unif. \\
\hline Pouteria sagotiana & 20 & 2 & 0,62 & 2,97 & 0,9 & Unif. & 20 & 2 & 0,76 & 3,04 & 0,9 & Unif. \\
\hline Sterculia speciosa & 60 & 6 & 0,12 & 3,80 & 0,7 & Unif. & 60 & 6 & 0,15 & 3,61 & 0,7 & Unif. \\
\hline Apeiba burchelli & 10 & 1 & 0,29 & 1,43 & 0,9 & Unif. & 10 & 1 & 0,33 & 1,39 & 0,9 & Unif. \\
\hline Attalea excelsa & 20 & 2 & 0,17 & 1,65 & 0,9 & Unif. & 20 & 2 & 0,19 & 1,57 & 0,9 & Unif. \\
\hline Mauritia flexuosa & 40 & 5 & 0,43 & 3,68 & 1,0 & Aleat. & 40 & 5 & 0,48 & 3,49 & 1,0 & Aleat. \\
\hline Socratea hexorriza & 20 & 4 & 0,06 & 1,57 & 1,8 & Agrup. & 20 & 4 & 0,06 & 1,46 & 1,8 & Agrup. \\
\hline Heisteria acuminata & $\mathrm{x}$ & $\mathrm{x}$ & $\mathrm{x}$ & $\mathrm{x}$ & $\mathrm{x}$ & $\mathrm{x}$ & 10 & 1 & 0,00 & 0,54 & 0,9 & Unif. \\
\hline Total & 2230 & 807 & 33,7 & 300 & & & 2330 & 926 & 38,7 & 300 & & \\
\hline
\end{tabular}

Tabela 4. Valores absolutos de freqüência, densidade, dominância e índice de McGuinnes (IGA) para as espécies no rio Mutuacá.

Table 4. Absolute values of frequency, density, dominance and McGuinnes (IGA) index for the species in the Mutuacá river.

\begin{tabular}{|c|c|c|c|c|c|c|c|c|c|c|c|c|}
\hline \multirow{2}{*}{ Espécies } & \multicolumn{6}{|c|}{ Novembro de 2000} & \multicolumn{6}{|c|}{ Novembro de 2005} \\
\hline & FA & DA & DoA & VI & IGA & COMP. & FA & DA & DoA & VI & IGA & COMP. \\
\hline Euterpe oleraceae & 100 & 344 & 9,15 & 65,07 & 7,5 & Cont. & 100 & 307 & 3,16 & 47,44 & 6,7 & Cont. \\
\hline Pentaclethra macroloba & 100 & 84 & 3,50 & 22,19 & 1,8 & Agrup. & 100 & 68 & 3,24 & 21,20 & 1,5 & Agrup. \\
\hline Virola surinamensis & 100 & 35 & 1,70 & 12,04 & 0,8 & Unif. & 100 & 36 & 2,02 & 13,94 & 0,8 & Unif. \\
\hline Terminalia guianensis & 70 & 18 & 2,67 & 11,91 & 1,5 & Agrup. & 70 & 18 & 3,03 & 13,89 & 1,5 & Agrup. \\
\hline Astrocarium murumuru & 100 & 39 & 0,67 & 9,59 & 0,8 & Unif. & 100 & 40 & 0,72 & 10,40 & 0,9 & Unif. \\
\hline Hura crepitans & 50 & 14 & 1,90 & 8,60 & 2,0 & Cont. & 50 & 14 & 2,55 & 11,23 & 2,0 & Cont. \\
\hline Carapa guianensis & 90 & 26 & 0,84 & 8,35 & 1,1 & Agrup. & 80 & 20 & 0,71 & 7,38 & 1,2 & Agrup. \\
\hline Pterocarpus amazonicus & 80 & 18 & 1,19 & 8,13 & 1,1 & Agrup. & 80 & 19 & 0,99 & 8,13 & 1,2 & Agrup. \\
\hline Miconia ceramicarpa & 70 & 41 & 0,45 & 8,08 & 3,4 & Cont. & 70 & 38 & 0,40 & 8,07 & 3,2 & Cont. \\
\hline Aspidosperma desmanthum & 60 & 12 & 1,36 & 7,24 & 1,3 & Agrup. & 60 & 13 & 1,45 & 8,13 & 1,4 & Agrup. \\
\hline Hevea brasiliensis & 90 & 18 & 0,68 & 7,07 & 0,8 & Unif. & 80 & 19 & 0,77 & 7,46 & 1,2 & Agrup. \\
\hline Cecropia palmata & 50 & 28 & 0,71 & 6,73 & 4,0 & Cont. & 60 & 23 & 0,62 & 6,69 & 2,5 & Cont. \\
\hline Tachigalia paniculata & 40 & 25 & 0,74 & 6,14 & 4,9 & Cont. & 40 & 23 & 0,96 & 6,99 & 4,5 & Cont. \\
\hline Guateria poeppigiana & 70 & 20 & 0,42 & 5,81 & 1,7 & Agrup. & 70 & 21 & 0,42 & 6,25 & 1,7 & Agrup. \\
\hline Swartzia cardiosperma & 80 & 13 & 0,51 & 5,70 & 0,8 & Unif. & 100 & 16 & 0,51 & 7,09 & 0,3 & Unif. \\
\hline Spondias mombin & 50 & 7 & 0,70 & 4,51 & 1,0 & Aleat. & 40 & 5 & 0,70 & 4,21 & 1,0 & Aleat. \\
\hline Tapirira guianensis & 10 & 1 & 0,02 & 0,52 & 0,9 & Unif. & 10 & 1 & 0,03 & 0,57 & 0,9 & Unif. \\
\hline Bombax munguba & 50 & 5 & 0,08 & 2,56 & 0,7 & Unif. & 40 & 4 & 0,11 & 2,28 & 0,8 & Unif. \\
\hline Matisia paraensis & 10 & 2 & 0,06 & 0,75 & 1,9 & Agrup. & 20 & 3 & 0,08 & 1,32 & 1,3 & Agrup. \\
\hline Pachira sp & 10 & 1 & 0,14 & 0,85 & 0,9 & Unif. & 10 & 2 & 0,23 & 1,29 & 1,9 & Agrup. \\
\hline Quararibea guianensis & 50 & 7 & 0,11 & 2,87 & 1,0 & Aleat. & 50 & 7 & 0,12 & 3,03 & 1,0 & Aleat. \\
\hline Protium spruceanum & 50 & 7 & 0,19 & 3,09 & 1,0 & Aleat. & 50 & 7 & 0,20 & 3,26 & 1,0 & Aleat. \\
\hline Campsiandra laurifolia & 10 & 1 & 0,04 & 0,57 & 0,9 & Unif. & 10 & 1 & 0,04 & 0,26 & 0,9 & Unif. \\
\hline Crudia oblonga & 30 & 4 & 0,05 & 1,66 & 1,1 & Agrup. & 20 & 3 & 0,05 & 1,25 & 1,3 & Agrup. \\
\hline Hymenaea oblongifolia & 10 & 1 & 0,00 & 0,48 & 0,9 & Unif. & $\mathrm{x}$ & $\mathrm{x}$ & $\mathrm{x}$ & $\mathrm{x}$ & $\mathrm{x}$ & Morreu \\
\hline Macrolobium augustifolium & 10 & 1 & 0,16 & 0,92 & 0,9 & Unif. & 10 & 1 & 0,19 & 1,06 & 0,9 & Unif. \\
\hline
\end{tabular}




\begin{tabular}{|c|c|c|c|c|c|c|c|c|c|c|c|c|}
\hline \multirow{2}{*}{ Espécies } & \multicolumn{5}{|c|}{ Novembro de 2000} & \multirow[b]{2}{*}{ COMP. } & \multicolumn{6}{|c|}{ Novembro de 2005} \\
\hline & FA & DA & DoA & VI & IGA & & FA & DA & DoA & VI & IGA & COMP. \\
\hline Mora paraensis & 30 & 3 & 0,04 & 1,52 & 0,8 & Unif. & 30 & 3 & 0,04 & 1,57 & 0,8 & Unif. \\
\hline Caryocar glabrum & 10 & 1 & 0,00 & 0,48 & 0,9 & Unif. & 10 & 1 & 0,00 & 0,50 & 0,9 & Unif. \\
\hline Licania heteromorpha & 20 & 2 & 0,02 & 0,99 & 0,9 & Unif. & 10 & 1 & 0,01 & 0,53 & 0,9 & Unif. \\
\hline Licania kunthiana & 10 & 1 & 0,00 & 0,48 & 0,9 & Unif. & 10 & 1 & 0,00 & 0,50 & 0,9 & Unif. \\
\hline Licania macrophylla & 10 & 1 & 0,02 & 0,53 & 0,9 & Unif. & 10 & 1 & 0,03 & 0,57 & 0,9 & Unif. \\
\hline Parinari excelsa & 20 & 2 & 0,63 & 2,70 & 0,9 & Unif. & 20 & 2 & 0,68 & 3,05 & 0,9 & Unif. \\
\hline Rheedia acuminata & 20 & 3 & 0,02 & 1,09 & 1,3 & Agrup. & 20 & 3 & 0,02 & 1,15 & 1,3 & Agrup. \\
\hline Symphonia globulifera & 30 & 4 & 0,16 & 1,95 & 1,1 & Agrup. & 20 & 3 & 0,20 & 1,69 & 1,3 & Agrup. \\
\hline Vismia macrophylla & 20 & 3 & 0,05 & 1,18 & 1,3 & Agrup. & $\mathrm{x}$ & $\mathrm{x}$ & $\mathrm{x}$ & $\mathrm{x}$ & $\mathrm{x}$ & Morreu \\
\hline Combretum cacoucia & 10 & 1 & 0,01 & 0,50 & 0,9 & Unif. & 10 & 1 & 0,01 & 0,52 & 0,9 & Unif. \\
\hline Manihot brachyloba & 10 & 1 & 0,00 & 0,48 & 0,9 & Unif. & 10 & 1 & 0,00 & 0,50 & 0,9 & Unif. \\
\hline Dipteryx sp & 20 & 2 & 0,07 & 1,14 & 0,9 & Unif. & 20 & 2 & 0,09 & 1,26 & 0,9 & Unif. \\
\hline Ormosia coutinhoi & 10 & 1 & 0,01 & 0,49 & 0,9 & Unif. & 20 & 2 & 0,02 & 1,02 & 0,9 & Unif. \\
\hline Platymiscium filipes & 60 & 10 & 0,49 & 4,61 & 1,1 & Agrup. & 60 & 9 & 0,55 & 4,92 & 1,0 & Aleat. \\
\hline Pterocarpus officinalis & 50 & 7 & 0,30 & 3,40 & 1,0 & Aleat. & 40 & 7 & 0,52 & 3,87 & 1,4 & Agrup. \\
\hline Vatairea guianensis & 10 & 1 & 0,27 & 1,23 & 0,9 & Unif. & 10 & 1 & 0,35 & 1,56 & 0,9 & Unif. \\
\hline Hernandia guianensis & 40 & 5 & 0,29 & 2,80 & 1,0 & Aleat. & 40 & 5 & 0,11 & 2,39 & 1,0 & Aleat. \\
\hline Saccoglotis guianensis & 20 & 2 & 0,02 & 1,01 & 0,9 & Unif. & 20 & 2 & 0,02 & 1,02 & 0,9 & Unif. \\
\hline Dendrobangia boliviana & 10 & 1 & 0,00 & 0,48 & 0,9 & Unif. & 20 & 2 & 0,01 & 0,99 & 0,9 & Unif. \\
\hline Aniba puchury-minor & 20 & 2 & 0,01 & 0,96 & 0,9 & Unif. & 20 & 3 & 0,07 & 1,29 & 1,3 & Agrup. \\
\hline Licaria cannella & 50 & 8 & 0,19 & 3,18 & 1,2 & Agrup. & 40 & 7 & 0,09 & 2,56 & 1,4 & Agrup. \\
\hline Licaria mahuba & 10 & 3 & 0,14 & 1,08 & 2,8 & Cont. & 20 & 3 & 0,30 & 1,99 & 1,3 & Agrup. \\
\hline Allantoma lineata & 30 & 4 & 0,35 & 2,49 & 1,1 & Agrup. & 20 & 4 & 0,47 & 2,62 & 1,8 & Agrup. \\
\hline Cedrela odorata & 20 & 2 & 0,07 & 1,14 & 0,9 & Unif. & 10 & 1 & 0,08 & 0,73 & 0,9 & Unif. \\
\hline Trichilia paraensis & 10 & 1 & 0,00 & 0,48 & 0,9 & Unif. & 10 & 1 & 0,01 & 0,51 & 0,9 & Unif. \\
\hline Trichilia surinamensis & 30 & 3 & 0,15 & 1,82 & 0,8 & Unif. & 20 & 2 & 0,15 & 1,42 & 0,9 & Unif. \\
\hline Inga $\mathrm{sp} 1$ & 40 & 12 & 0,52 & 4,16 & 2,3 & Cont. & 50 & 13 & 0,69 & 5,43 & 1,9 & Agrup. \\
\hline Inga $\mathrm{sp} 2$ & 10 & 1 & 0,01 & 0,49 & 0,9 & Unif. & 10 & 1 & 0,01 & 0,51 & 0,9 & Unif. \\
\hline Inga sp3 & 30 & 3 & 0,04 & 1,51 & 0,8 & Unif. & 20 & 2 & 0,02 & 1,04 & 0,9 & Unif. \\
\hline Inga cinnamomea & 20 & 2 & 0,01 & 0,97 & 0,9 & Unif. & 10 & 1 & 0,01 & 0,53 & 0,9 & Unif. \\
\hline Pithecellobium $\mathrm{sp}$ & 70 & 11 & 0,09 & 3,95 & 0,9 & Unif. & 80 & 14 & 0,12 & 4,92 & 0,9 & Unif. \\
\hline Ficus maxima & 30 & 10 & 0,18 & 2,63 & 2,8 & Cont. & 30 & 10 & 0,79 & 4,64 & 2,8 & Cont. \\
\hline Ficus pertusa & 20 & 7 & 0,40 & 2,58 & 3,1 & Cont. & 20 & 6 & 0,59 & 3,21 & 2,7 & Cont. \\
\hline Olmedia caloneura & 20 & 2 & 0,28 & 1,73 & 0,9 & Unif. & 20 & 2 & 0,33 & 1,99 & 0,9 & Unif. \\
\hline Calyptranthes speciosa & 10 & 2 & 0,01 & 0,59 & 1,9 & Agrup. & 10 & 1 & 0,02 & 0,54 & 0,9 & Unif. \\
\hline Eugenia browsnbergii & 50 & 5 & 0,02 & 2,40 & 0,7 & Unif. & 40 & 5 & 0,01 & 2,08 & 1,0 & Aleat. \\
\hline $\begin{array}{l}\text { Heisteria acuminata } \\
\text { Callycophyllum }\end{array}$ & 10 & 1 & 0,00 & 0,47 & 0,9 & Unif. & 20 & 2 & 0,00 & 0,98 & 0,9 & Unif. \\
\hline spruceanum & 10 & 3 & 0,21 & 1,27 & 2,8 & Cont. & 10 & 3 & 0,00 & 0,71 & 2,8 & Cont. \\
\hline Metrodorea flavida & 60 & 10 & 0,06 & 3,40 & 1,1 & Agrup. & 50 & 11 & 0,29 & 3,99 & 1,6 & Agrup. \\
\hline Crysophyllum excelsum & 60 & 8 & 0,11 & 3,33 & 0,9 & Unif. & 50 & 8 & 0,10 & 3,06 & 1,2 & Agrup. \\
\hline Pouteria sagotiana & 40 & 5 & 0,09 & 2,23 & 1,0 & Aleat. & 40 & 5 & 0,13 & 2,47 & 1,0 & Aleat. \\
\hline Spdesc & 30 & 4 & 0,08 & 1,73 & 1,1 & Agrup. & 30 & 6 & 0,09 & 2,07 & 1,7 & Agrup. \\
\hline Guazuma ulmifolia & 40 & 4 & 0,72 & 3,88 & 0,8 & Unif. & 20 & 3 & 0,52 & 2,68 & 1,3 & Agrup. \\
\hline Herrania mariae & 20 & 2 & 0,01 & 0,95 & 0,9 & Unif. & 20 & 4 & 0,01 & 1,23 & 1,8 & Agrup. \\
\hline Sterculia speciosa & 20 & 3 & 0,08 & 1,26 & 1,3 & Agrup. & 20 & 3 & 0,11 & 1,41 & 1,3 & Agrup. \\
\hline Theobroma cacao & 10 & 1 & 0,01 & 0,48 & 0,9 & Unif. & 10 & 1 & 0,01 & 0,51 & 0,9 & Unif. \\
\hline Apeiba burchelii & 30 & 3 & 0,29 & 2,22 & 0,8 & Unif. & 30 & 4 & 0,33 & 2,59 & 1,1 & Agrup. \\
\hline
\end{tabular}

FLORESTA, Curitiba, PR, v. 37, n. 3, set./dez. 2007. 


\begin{tabular}{|c|c|c|c|c|c|c|c|c|c|c|c|c|}
\hline \multirow{2}{*}{ Espécies } & \multicolumn{6}{|c|}{ Novembro de 2000} & \multicolumn{6}{|c|}{ Novembro de 2005} \\
\hline & FA & DA & DoA & VI & IGA & COMP. & FA & DA & DoA & VI & IGA & COMP. \\
\hline Attalea excelsa & 20 & 3 & 0,28 & 1,83 & 1,3 & Agrup. & 20 & 2 & 0,24 & 1,70 & 0,9 & Unif. \\
\hline Manicaria saccifera & 40 & 9 & 0,74 & 4,46 & 1,8 & Agrup. & 40 & 9 & 0,98 & 5,51 & 1,8 & Agrup. \\
\hline Oenocarpus bacaba & 30 & 4 & 0,11 & 1,83 & 1,1 & Agrup. & 30 & 4 & 0,13 & 1,96 & 1,1 & Agrup. \\
\hline Tachigalia myrmecophila & $\mathrm{x}$ & $\mathrm{x}$ & $\mathrm{x}$ & $\mathrm{x}$ & $\mathrm{x}$ & $\mathrm{x}$ & 10 & 1 & 0,04 & 0,60 & 0,9 & Unif. \\
\hline Ocotea $\mathrm{sp}$ & $\mathrm{x}$ & $\mathrm{x}$ & $\mathrm{x}$ & $\mathrm{x}$ & $\mathrm{x}$ & $\mathrm{x}$ & 10 & 1 & 0,00 & 0,50 & 0,9 & Unif. \\
\hline Mouriri acutiflora & $\mathrm{x}$ & $\mathrm{x}$ & $\mathrm{x}$ & $\mathrm{x}$ & $\mathrm{x}$ & $\mathrm{x}$ & 10 & 1 & 0,00 & 0,49 & 0,9 & Unif. \\
\hline Pithecellobium inaequale & $\mathrm{x}$ & $\mathrm{x}$ & $\mathrm{x}$ & $\mathrm{x}$ & $\mathrm{x}$ & $\mathrm{x}$ & 10 & 1 & 0,00 & 0,49 & 0,9 & Unif. \\
\hline Apeiba burchelii & $\mathrm{x}$ & $\mathrm{x}$ & $\mathrm{x}$ & $\mathrm{x}$ & $\mathrm{x}$ & $\mathrm{x}$ & 30 & 4 & 0,33 & 2,59 & 1,1 & Agrup. \\
\hline Total & 2740 & 961 & 35,7 & 300 & & & 2661 & 903 & 32,7 & 300 & & \\
\hline
\end{tabular}

FA: Freqüência absoluta; DA: Densidade absoluta; DoA: Dominância absoluta; VI: Valor de Importância; IGA: Índice de Mc Guinnes; COMP.: Comportamento; Cont.: Espécies com distribuição contagiosa; Agrup.: Espécies com tendência ao agrupamento; Unif.: Espécies com distribuição uniforme; Aleat.: Espécies com tendência de distribuição aleatória.

No rio Mutuacá, a extração seletiva de palmito e de madeira influenciou bastante na redução da densidade, pois muitas árvores morreram em conseqüência da queda das que foram extraídas. As espécies que sofreram redução de densidade mais significativa foram: Euterpe oleracea, $37(10,8 \%)$; Pentaclethra macroloba, 16 (19,0\%); Carapa guianensis, 6 (23,1\%); e Cecropia palmata, 5 (17,9\%).

Em relação às quatro espécies de maior valor de importância (VI) nas áreas em estudo - Euterpe oleracea, Astrocaryum murumuru, Mora paraensis e Pentaclethra macroloba - Almeida (2004), em estudos botânicos realizados em Cajuúna (Afuá/PA), usando DAP mínimo de $10 \mathrm{~cm}$, encontrou resultados semelhantes.

Em relação às seis espécies de maior valor de importância (VI) nas áreas em estudo - Euterpe oleracea, Astrocaryum murumuru, Mora paraensis, Pentaclethra macroloba, Carapa guianensis e Virola surinamensis - Jardim et al. (2004), em estudos de florística e estrutura de floresta de várzea em seis localidades do município de Breves (PA), usando DAP mínimo de $20 \mathrm{~cm}$ para as dicotiledôneas e de 10 $\mathrm{cm}$ para as palmeiras, encontrou resultados semelhantes em algumas localidades, como foi o caso de Laranjal II, e resultados aproximados dos encontrados no presente trabalho, em outras, como foi o caso de Laranjal I, onde Mora paraensis e Carapa guianensis apresentaram VI baixo; Furo do Alambique, onde Virola surinamensis e Carapa guianensis apresentaram VI baixo; Macena, com Euterpe oleracea e Astrocaryum murumuru de VI baixo; Pracaxi-Açú, com Carapa guianensis de VI baixo e Pentaclethra macroloba ausente; e Mapuá, onde Astrocaryum murumuru esteve ausente.

\section{CONCLUSÕES}

As espécies açaí (Euterpe oleraceae Mart.), pracaxi (Pentaclethra macroloba O. Kuntze), murumuru (Astrocaryum murumuru Mart.) e andiroba (Carapa guianensis Aubl.) ocorrem tanto na várzea alta quanto na várzea baixa, com elevado valor de importância (VI), mostrando boa capacidade de adaptação aos dois ambientes.

A espécie pracuúba (Mora paraensis Ducke), embora ocorra na várzea baixa, mostrou melhor adaptação à várzea alta, onde assumiu elevado valor de importância (VI).

A espécie assacu (Hura crepitans L.) monstrou melhor adaptação à várzea baixa, onde apresentou alto valor de importância (VI) e total ausência na várzea alta.

Clima tropical, solos hidromórficos de boa fertilidade e o regime de cheias e vazantes do rio Amazonas, o qual, em vários períodos do ano, inunda a floresta, fazem com que o ambiente de várzea estuarina crie uma condição especial, tornando altamente dinâmico. As árvores instaladas sobre sedimentos recentes, mesmo aquelas com enormes sapopemas, não escapam do tombo. As clareiras então abertas, mais as condições citadas anteriormente, permitem a germinação de sementes e o rápido desenvolvimento das plantas.

O tipo de relacionamento e uso que o extrativista ribeirinho faz da propriedade florestal têm forte influência sobre a estrutura e dinâmica observada entre as espécies que ocorrem na área. 
No Furo do Mazagão, espécies de valor comercial madeireiro como Mora paraensis, mesmo com toda a pressão de uso da madeira, manteve a população, razoavelmente elevada, existente anteriormente; Licania macrophilla, mesmo sendo portadora de uma copa com capacidade de dificultar a produção de fruto de açaí, também foi mantida na área, e Astrocaryum murumuru, mesmo sendo considerada uma espécie sem valor comercial e que também exerce grande competição com os açaizeiros, teve sua densidade aumentada.

No rio Maniva, espécies de valor madeireiro como Mora paraensis, mesmo apresentando população elevada (80 árvores), foi mantida na área, e Astrocaryum murumuru, mesmo com sua população bastante elevada, teve mantidas as suas 141 palmeiras.

No rio Mutuacá, em razão de intervenções implementadas pelo proprietário, para retirada de madeira e de palmito, os ingressos mais significativos ficaram por conta de espécies do grupo das leguminosas, plantas com grande capacidade de dispersão, haja vista a capacidade de produção de sementes e de estabelecimento das mudas, como foi o caso de Pentaclethra macroloba, Pterocarpus amazonicus e Swartzia cardiosperma.

\section{REFERÊNCIAS}

ALMEIDA, S. S.; AMARAL, D. D. do; SILVA, A. S. L. da. Análise florística e estrutura de florestas de várzea no estuário amazônico. Acta Amazônica, Manaus, v. 34, n. 4, p. 513-524, 2004.

AMAPÁ. Instituto de Pesquisas Científicas e Tecnológicas do Estado do Amapá. Zoneamento ecológico econômico da área sul do estado do Amapá - ATLAS. Macapá: IEPA/GEA/AP, 2000.

AMAPÁ. Instituto de Pesquisas Científicas e Tecnológicas do Estado do Amapá. Macrodiagnóstico do estado do Amapá: primeira aproximação do zoneamento ecológico econômico. Macapá: IEPA - ZEE, 2002. $140 \mathrm{p}$.

ANDERSON, A. B.; GELY, A.; STRUDWICK, J.; SOBEL, G. L.; PINTO, M. C. Um sistema agroflorestal na várzea do estuário amazônico (Ilha das Onças, município de Barcarena, estado do Pará). Acta Amazônica, Manaus, v. 15, n. 1-2, p. 195-224, 1985. (Suplemento).

BARROS, P. L. C. de; MACHADO, S. do A. Aplicação de índices de dispersão em espécies de florestas tropicais da Amazônia brasileira. Curitiba. FUPEF/UFPR, 1984. 44 p. (FUPEF. Série Científica, n. 1).

BARROS, A. C.; UHL, C. Logging along the Amazon River and estuary: Patterns, problems and potential. Forest Ecology and Management, Amsterdam, v. 77, p. 87-105, 1995.

CURTIS, J. T. The vegetation of Wisconsin. An ordenation of plant communities. Madison: University of Wisconsin Press, 1959. 657 p.

CURTIS, J. T.; McINTOSH, R. P. The interrelations of certain analytic and synthetic phytosociological characters. Ecology, Tempe, v. 31, n. 3, p. 434-50, 1950.

DUCKE, A.; BLACK, G. A. Notas sobre a fitogeografia da Amazônia Brasileira. Belém: Instituto Agronômico do Norte, 1954. 62 p. (Boletim Técnico, n. 29).

FALESI, I. C.; SILVA, B. N. R. da Ecossistemas de várzeas da região do Baixo Amazonas. Belém: Embrapa Amazônia Oriental, 1999. 75 p.

HIRAOKA, M. Miriti (Mauritia flexuosa) palms and their uses and management among the ribeirinhos of the Amazon Estuary. In: PADOCH, C.; AYRES, J. M.; PINEDO-VASQUEZ, M.; HENDERSON, H. Diversity, development, and conservation of Amazonia's whitewater floodplains. New York. The New York Botanical Garden Press, 1999. p. 169-186.

HOSOKAWA, R. T. Manejo e economia de florestas. Roma: FAO/ONU, 1986. 125 p.

JARDiM, M. A. G. AMARAL, D. D.; SANTOS, G. C. dos; MEDEIROS, T. D. S.; SilvA, C. A.; FRANCEZ, D. da C.; COSTA NETO, S. V. da. Análise florística e estrutural para avaliação da 
fragmentação nas florestas de várzea do estuário amazônico. In: JARDIM, M. A. G.; MOURÃO, L.; GROISSMAN, M. Açaí: possibilidades e limites para o desenvolvimento sustentável no estuário amazônico. Belém: Museu Paraense Emílio Goeldi, 2004. p. 101-121.

MORÁN, E. F. A ecologia das populações da Amazônia. Petrópolis: Vozes, 1990. 367 p.

MUELLER-DOMBOIS, D.; ELLENBERG, H. Aims and methods of vegetation ecology. New York: J. Wiley \& Sons, 1974. 547 p.

NOGUEIRA, O. L. Estrutura e dinâmica populacional de açaizais nativos de várzea na região do Baixo Tocantins, estado do Pará. Belém: Embrapa Amazônia Oriental, 1999. 21 p. (Boletim de Pesquisa, n. 15).

QUEIROZ, J. A. L. de. Fitossociologia e distribuição diamétrica em floresta de várzea do estuário do rio Amazonas no estado do Amapá, 2004. 101 f. Dissertação (Mestrado em Ciências Florestais) - Setor de Ciências Agrárias, Universidade Federal do Paraná, Curitiba, 2004.

QUEIROZ, J. A. L. de; MOCHIUTTI, S. Tipos de açaizais do Estuário Amazônico e efeitos das intervenções praticadas por extratores ribeirinhos. In: SABOGAL, C.; SILVA, J. N. M. (Orgs.). Manejo integrado de florestas úmidas neotropicais por indústrias e comunidades: aplicando resultados de pesquisa, envolvendo atores e definindo políticas públicas. Belém: CIFOR/Embrapa Amazônia Oriental, 2002, p. 344-350.

RABELO, B. V. (Coord.). Mazagão: realidades que devem ser conhecidas. Macapá: Instituto de Pesquisas Científicas e Tecnológicas do Estado do Amapá-IEPA, 2005. 119 p.

RABELO, F. G. Composição florística, estrutura e regeneração de ecossistemas florestais na região estuarina do rio Amazonas-Amapá-Brasil. 72 p. Dissertação (Mestrado em Ciências Florestais) Faculdade de Ciências Agrárias do Pará, Belém, 1999.

VALENTE, M. A.; OLIVEIRA JÚNIOR, R. C.; RODRIGUES, T. E.; SANTOS, P. L.; SILVA, J. M. L.; CARDOSO JÚNIOR, E. Q. Solos da ilha de Santana, município de Santana, estado do Amapá. Belém: Embrapa CPATU, 1998. 34 p. (Documentos, n.138).

VASQUEZ, M. P.; RABELO, F. G. Sustainable management of an Amazonian Forest for timber production: a myth or reality? Plec News and Views, [S.1.], n. 12, p. 20-28, 1999. 